\title{
Interactive comment on "On the influence of sea-ice physics in multi-decadal ocean-ice hindcasts" by Petteri Uotila et al.
}

\section{Anonymous Referee \#2}

Received and published: 2 November 2016

\section{General comments}

The paper presents an evaluation of the updates to the LIM sea ice model, within the NEMO ocean model, version 3.6. A comparison is made between the previous configuration, LIM2, and the new one, LIM3. Some sensitivity runs are performed, to allow differences between LIM2 and LIM3 to be attributed to particular configuration changes. The model output is also evaluated against appropriate observational datasets. Only the sea ice configuration, and not that of the ocean, has changed between the two model versions, but the authors carry out a detailed study of the impact on the ocean as well as the sea ice. The paper is well-structured and methodical. It is generally scientifically robust, although I have some specific concerns which I will outline below. The paper is well-written, and the standard of English is generally good. I recommend

Printer-friendly version

Discussion paper 
publication after the issues outlined below have been addressed.

\section{Specific comments}

- Five model configurations are analysed in the paper - LIM2, LIM3, LIM3MC, and the two simulations to test sensitivity to the freshwater adjustments. The configurations are described in detail in Sections 2.4, 4.1 and 4.2. However, I think that a useful complement to this would be a table summarising the settings in each configuration. That way, the reader could refer back easily to the table while studying the results.

- In several figures, the authors use both green and red. This should be avoided if possible, as readers who are colour-blind will have difficulty in distinguishing between these colours. The "rainbow" colour scheme used in Figure 3 should be avoided for the same reason if possible.

- The atmospheric forcing dataset used by the authors is based on ERA-40 before 2001, and ERA-Interim thereafter. Several authors have cast doubt on the reliability of ERA-40 in the polar regions, due to the sparsity of observations there. For example, Screen Simmonds (2011) noted a discontinuity in Arctic temperature in 1997, leading to a significantly exaggerated warming in the mid-to-lower troposphere. While the main period considered by the authors, 2003-2012, is wholly covered by the ERA-Interim-based forcing, the period immediately before could be affected by the inaccuracies in ERA-40, and there could be some residual impact in 2003-2012 due to the "memory" of the ocean. In addition, the authors do sometimes make use of the model output from before 2003 (for example, when discussing the trends in ice extent since 1979 as presented in Figure 2b). They should therefore reflect in the paper on the possible impact on the results of any inaccuracies in the ERA-40 forcing data.

Printer-friendly version

Discussion paper 
- In Section 3.1, where the authors refer to the trends in SH ice extent as "statistically significantly increasing", they should specify the level of significance and the method used to assess it. In a number of other places in the sea ice sections, the authors refer to results as "significant". For example, in Section 3.2, the interannual variations in annual mean $\mathrm{NH}$ ice volume are "significant". In the same section, the LIM3 SH ice volume is said to have a "significant positive trend", the trend in GIOMAS ice volume is "significantly negative", and LIM2 has "no significant trend". There are further examples in the discussion of the sensitivity runs in Section 4. Do the authors mean "statistically significant"? And if so, how was this assessed and what is the level of significance? This should be specified in the paper.

- In the discussion of the impacts on the ocean (Section 5), there is no discussion of statistical significance of the results. In a number of cases, I wondered if the inter-model differences, and the differences with respect to observations, were significant with respect to the interannual variability, especially where the differences were small. For example, in Section 5.6, the authors refer to "small temperature and salinity differences" between the LIM simulations. And in Section 5.8, they refer to the fact that the AMOC in LIM2 is up to $0.4 \mathrm{~Sv}$ stronger than that in LIM3. Are these differences statistically significant above the interannual variability?

- In most cases, the authors restrict their analysis to the last decade of the 54-year simulations. Given that the model starts from rest and will take some time to spin up, this is the correct approach. However, restricting the period of study to 10 years means that multidecadal variability will not be picked up in the analysis. To what extent are the differences between LIM2 and LIM3, or the differences between the LIM configurations and observations, dependent on factors that may be subject to multidecadal variability? I appreciate it may be difficult to answer this question with the results available, but I think the authors should at least

Printer-friendly version

Discussion paper
Interactive comment

\section{C3}


mention it in the discussion of their results.

- In Section 3.2 (page 10, lines 12-13), the authors state "Close to the ice edge, LIM3 has a smaller ice extent and a lower ice concentration at regions". I'm not sure what is meant by "at regions". Does it mean "in certain locations"? If so, then please change it.

- In the conclusions, the authors refer to the new sea ice albedo scheme implemented in LIM3 in April 2016. They state "Our preliminary tests on this new scheme demonstrate the robustness of LIM3, as its sea-ice distribution appears almost insensitive to changes in summer albedo". I find this statement surprising, as several studies have shown that sea ice simulations are indeed extremely sensitive to albedo in summer (see, e.g., Kim et al., 2006, and Rae et al., 2014). While use of a reliable atmospheric forcing dataset should reduce the need for tuning via albedo adjustments (see, e.g., Hunke, 2010), I would still expect the sea ice simulation to be sensitive to albedo changes. Perhaps I have misunderstood what the authors mean by this statement, but I would appreciate some clarification.

- In the figures, the results are generally presented as LIM3-LIM2, or LIM3-obs. This makes sense, as the authors are presenting the first study of a simulation with the new LIM3 configuration, and comparing it with the previous configuration (LIM2) and observations. However, in the text the authors often discuss the results in reverse. For example, on page 16, line 3, when referring to Figure 9e: "In the Southern Ocean, the observed mixed layer is shallower than the LIM3 mixed layer...". This wording seems a bit strange and "back-to-front" to me, as the results in the figure are presented as LIM3 minus obs (and anyway the purpose of the paper is to assess LIM3 against observations, not the other way round). I would prefer to see the wording in the text reflect the way the results are presented in the figures ("In the Southern Ocean, the LIM3 mixed layer is deeper

Printer-friendly version

Discussion paper 
than observed"), unless there is a good reason to do otherwise. There are several other examples of this type of "back-to-front" wording throughout the paper to which similar remarks apply.

\section{Technical corrections}

- Where the authors mention results without including figures (e.g. in Section 3.2, although there are other places where this occurs), it would be useful for the reader if they stated "not shown here".

- Page 7, line 5: "Figures 2a and b" should be "Figures 2a and c".

- Page 7, line 14: "minimum extends" should be "minimum extents".

- Page 9, line 15: "a lesser significance as in the..." should be "a lesser significance than in the...."

- Page 14, line 4: "smaller between LIM3 and WOA13 than between PHC3" should be "smaller between LIM3 and WOA13 than between LIM3 and PHC3"

- Page 14, line 21: "around the East Antarctica" should be "around the East Antarctic" or "around East Antarctica" (without "the").

- Page 14, line 30: "loose heat to the water masses" should be "lose heat to the water masses".

- Page 15, line 2: "might be associated to" should be "might be associated with".

Printer-friendly version

Discussion paper

- Page 15, line 33: "This is at least partly due to cold, non-responsive and prescribed winter atmosphere..." should be "This is at least partly due to the cold, non-responsive prescribed winter atmosphere..." (add "the", delete "and"). 
- Page 16, line 10: "and one where denser LIM2 surface entrain deeper" should be "and one where the denser LIM2 surface entrains more deeply".

- Page 16, line 11: "with most distinct ones visible" should be "with the most distinct ones visible".

- Page 16, line 18: "Danabasoglu et al. (2014) assessed the mean AMOC of eighteen ocean-ice models forced by prescribed atmospheric forcing from 19482007 and run five repetitive forcing cycles...": Inconsistent tenses (past/present). "run" should be "ran".

- Page 16, line 20: "Here, results of..." should be "Here, the results of...".

\section{References}

Hunke, E.C., 2010. Thickness sensitivities in the CICE sea ice model. Ocean Modell. 34, 137-149. http://dx.doi.org/10.1016/j.ocemod.2010.05.004.

Kim, J.G., Hunke, E.C., and Lipscomb, W.H., 2006. Sensitivity analysis and parameter tuning scheme for global sea-ice modeling. Ocean Modell. 14, 61-80. http://dx.doi.org/10.1016/j.ocemod.2006.03.003.

Rae, J.G.L., Hewitt, H.T., Keen, A.B., Ridley, J.K. Edwards, J.M., and Harris, C.M., 2014. A sensitivity study of the sea ice simulation in the global coupled climate model, HadGEM3. Ocean Modell. 74, 60âĂŤT6. http://dx.doi.org/10.1016/j.ocemod.2013.12.003.

Screen, J.A., and Simmonds, I., 2011. Erroneous Arctic Temperature Trends in the ERA-40 Reanalysis: A Closer Look. J. Climate, 24. 2620-2627. doi: 10.1175/2010JCLI4054.1. 
Interactive comment on Geosci. Model Dev. Discuss., doi:10.5194/gmd-2016-187, 2016.

GMDD

Interactive

comment 